\title{
Density functional theory on the polymerization of diaminofluorene
}

\author{
Fuad A. Asswadi*, Ali H. Aldhamri, Fathy A. Abeed \\ Laboratory of Electrochemistry, Chemistry Department, Faculty of Science, Ibb University, Ibb, Yemen
}

\section{Email address:}

fuad19782002@yahoo.com

\section{To cite this article:}

Fuad A. Asswadi, Ali H. Aldhamri, Fathy A. Abeed. Density Functional Theory on the Polymerization of Diaminofluorene. Science Journal of Chemistry. Vol. 3, No. 2, 2015, pp. 18-22. doi: 10.11648/j.sjc.20150302.11

\begin{abstract}
Density- functional theory (DFT) calculations on 2,7-diaminofluorene in order to understand the various possible mechanisms of growth, there are two possible mechanisms on it which involve the coupling of cationic radicals with another cationic radical or with a neutral oligomer. The electrochemical properties of this oligomer is understood, but until now there is very little known about the nature of the intermediates and the transition states leading to polymerization. The initial oxidation, forming stable intermediates, releasing protons and further oxidations are studied in terms of the charges distributions, energies changes in the geometry and vibrational frequencies have been calculated using DFT (B3LYP). Dramatic differences have been observed in the infrared band intensities of the cations compared to their neutral parents. Hydrogen bonding between DAF molecules in the crystal structure the direct comparison of theoretical calculations with experiment, so Hartree-Fock (HF) and Becke-Perdew density-functional theory (DFT) calculations are used as benchmarks for the semiempirical and molecular mechanics results.
\end{abstract}

Keywords: DFT, HF, Mechanism, Oligofluorene

\section{Introduction}

Electrically conducting organic polymers consisting of chains with alternating single and double bonds (conjugated polymers) are very important materials because of their wide range of applications in batteries, biosensors, coating material, for metals, photoresists, optical switches. LED devices, electrochromic and electroluminescent devices (1). Most of these polymers are insoluble or less soluble in common solvents and their characterization is difficult. Among these conducting polymers polypyrroles and polythiophenes have drawn more attention due to their very wide range application areas(2-7).

Many aromatic amines are known to be, or suspected to be, carcinogens. These aromatic amines are present in barbecued meats, tobacco smoke, automobile exhaust and dyes. A great deal of research over the past several decades has focused on 2-(acetylamino)fluorene (AAF), a potent mutagen and a prototypical example of the mutagenic aromatic amines. Theoretical calculations of these materials helped to the understanding of their formation mechanisms and made the synthesis of the materials with desired properties possible $(8,9)$

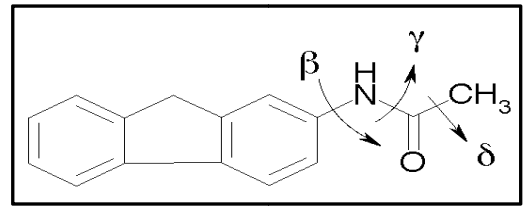

Figure 1. 2-(acetylamino)fluorene (AAF). The dihedral angles are shown.

First predicted in 1931 by Go ppert-Mayer1 and then observed in 1961 by Kaiser and Garrett,2 two-photon absorption phenomena are of a great interest for new technologies. 3 Several strategies have since been developed for creating new molecular materials exhibiting strong twophoton absorptions(10-12), and the synthesis of conjugated organic molecular compounds is one of them.4,5 However, the two-photon absorption properties of most of these molecular organic compounds are based on intramolecular charge transfers (donor-acceptor or substituted strategy), $6,7,8,9,10$ which makes the most efficient ones colored and then incompatible with some specific applications in the visible region(13-15).

In recent years, research in heterocyclic conducting polymers has received a great deal of attention due to the possibility to fine-tune their optical and electronic 
properties via chemical modification of their structures, in addition to the ease in chemical and electrochemical synthesis (16-20). Several electro-polymerization kinetic models have been proposed. Del-Valle et al. proposed a radical cation poly-condensation mechanism in which different stages simultaneously occur making the mechanism quite complicate (21-26)

The present work encloses a theoretical study on diaminofluorene oligomers using Density Functional Theory (DFT). DFT is very attractive in calculations involving finite systems because even the lowest level of DFT. Density functional theory (DFT) method had successfully been used to study band gaps of conjugated organic polymers where the HOMO/LUMO difference provide good estimate of the excitation energy. While there is some controversy surrounding the interpretation of DFT. orbitals energies, there has been found that HOMO/LUMO energy difference offers a very good estimate of band gaps $(13,27)$. The purpose in choosing diaminofluorene substituents is founded on their electro-donning nature which could possibly play in favor of the reactivity of the aromatic rings, facilitating in this way the synthesis of fluorene-based polymers. In this study, we also employ the extrapolation approach in order get some property of the infinite chain length polymer. This is a reliable method, since for most polymers, the properties of their small oligomers are already well known $(10,28-30)$. The aim of the paper is to compare the effects that have different substituents in different positions within the oligomer chain on its electrical conducting properties.

\section{Computational Details}

All calculations on the oligomers studied in this work were performed using Gaussian program package (15). The geometries of neutral and doubly charged oligomers containing 2, 4, 6,7,8 and 9 rings were fully optimized with DFT using the three-parameter compound of Becke B3LYP (16) together with the basis set $3-21 G^{*}(17,31)$. The geometry of the aminofluorene monomers and the aminofluorene monomers substituted with another radical groups of fluorene were fully optimized in their ground and charged states at the same level of theory. The ionization potentials (IP) were calculated as the differences of the energies between the cation and the neutral molecules. These IPs were calculated from the optimized ground-state geometries. The lowest transition energies dominated by HOMO-LUMOp-p* transitions, or band gaps (Eg), of the oligomers were estimated by the energy difference between the highest occupied molecular orbital (HOMO) and the lowest unoccupied molecular orbital (LUMO) (18). The calculations were carried out on oligofluorene containing 2 , $4,6,7,8$ and 9 rings substituted by radical cation of aminofluorenegroups with an alternated head-to-head (HH) and tail-totail (TT) coupling (19) (see Fig.) and a stagepolymerization.

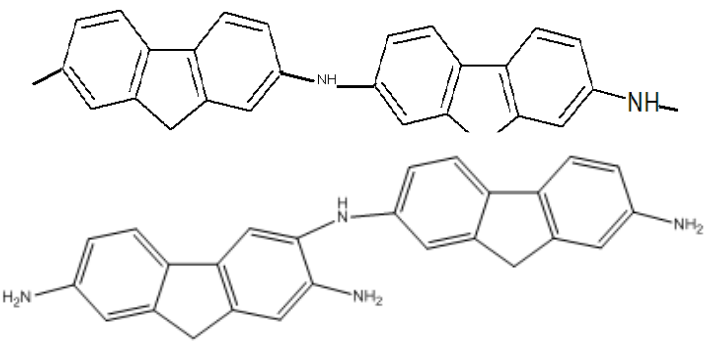

Figure 2. Dimmer of DAF monomer structure.

Table 1. Energy of substituted aminofluorene monomers.

\begin{tabular}{ll}
\hline Monomer & Energy(Kcal/mol $)$ \\
\hline DAF & -382003.545205386 \\
DAFR2 & -381596.785818768 \\
DAFR3 & -381572.976583344 \\
DAFR4 & -381571.784816352 \\
\hline
\end{tabular}

Table 2. The eigenvalues of HOMO and LUMO and energy gap of HOMO and $L U M O$.

\begin{tabular}{llll}
\hline Monomer & HOMO & LUMO & $\Delta \mathbf{E}$ ev \\
\hline 2,7DAF & -0.20295 & 0.00974 & -5.78772 \\
R2 & -0.21838 & -0.0068 & -5.75751 \\
R3 & -0.20602 & 0.0083 & -5.83208 \\
R4 & -0.20734 & 0.00529 & -5.78609 \\
\hline
\end{tabular}

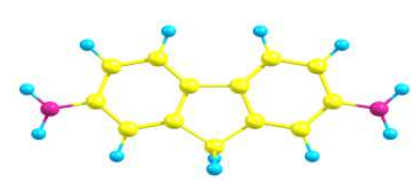

DAF

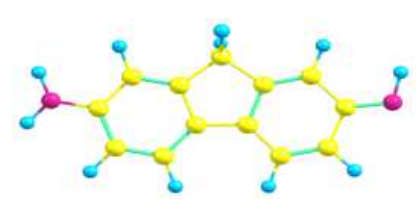

$\mathrm{R} 2$

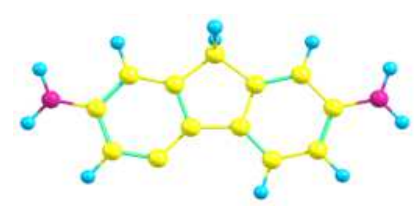

R3

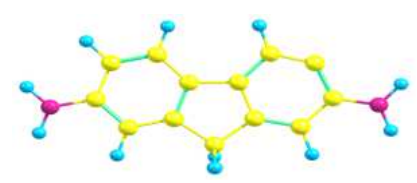

R4

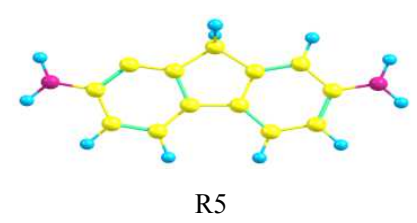

Figure 3. DAF monomer and different radicals of 2,7-Diaminofluorene 


\section{Results and Discussion}

According to the results shown in Tables 1,2, the substituted radical monomers decrease the energy from R1 to $\mathrm{R} 1,1$. These results are in line with the idea that the fluorenebased substituted polymers could be obtained with less difficulty than the non-substituted polymer. The resulting calculations of energy, HOMO, LUMO and $\triangle \mathrm{E}$ values of non-substituted fluorene oligomers are shown in Table 2. the molecular stability increases and comes accompanied by a decrease in the values of IPs. The energy of molecular orbitals HOMO and LUMO decrease also as the oligomer grew up in size, which brings as consequence a decrease of $\Delta \mathrm{E}$ values. these low values indicate improvement in conductivity which favors electron mobility, they indicate as well a higher reactivity of the molecules with a possible drop-off in stability which would difficult its synthesis. The results of energy HOMO, LUMO and $\triangle \mathrm{E}$ values of the substituted oligomers are shown in Table 2. The energy values follow the general trend to decrease as the oligomer chain extends due to the presence of a greater number of molecular orbitals which allow lower molecular energies. It was found that the substituted oligomers possess lower energy values than the corresponding non-substituted oligomers. The overall energetic stability trend found was $\mathrm{R} 3>\mathrm{R} 4>\mathrm{R} 2$, where the individual differences were not significant. These numerical small differences should not be overstated and must be looked upon with caution, because in extending the size of the polymer chain they might become significant. The linear regressions clearly indicate that increasing the size of radical-substituted with another radical monomer at these positions leads to decreasing the value of $\Delta \mathrm{E}$. This tendency is due to the fact that head to tail has the greatest planarity and it has been shown that this topological feature favors the conductivity. The energetic and the electronic properties of the substituted aminofluorene oligomers are show there are no significant differences in energy between equal size oligomers. The stability tendency found was R3 $>$ R4 $>$ R2. On the other hand, the energy needed of the substituted at $\mathrm{R} 1,1$ position is less than on another sites of fluorene oligomer and the nitrogen is less electronegative than the hydrogen therefore it has less difficulty in stabilizing a partially positive charge. Hence the dicationic states are more stable than the dianionic states. In general the $\mathrm{E}$ values of the radical-substituted on $\mathrm{R} 3$ or $\mathrm{R} 4$ are higher than the radical-substituted on R2 there are show in table. 1 .

Table 3. Energies of aminofluorene and different cation of fluorine.

\begin{tabular}{llllll}
\hline & & $\mathbf{E}_{\mathbf{0}}$ & $\mathbf{E}_{\mathbf{T}}$ & H enthalpy & G \\
\hline 2,7-DAF & & -608.54 & -608.527 & -608.52635 & -608.577 \\
DAF & R1 & -607.865 & -607.852 & -607.85147 & -607.903 \\
DAF & R2 & -607.904 & -607.893 & -607.89166 & -607.942 \\
DAF & R3 & -607.866 & -607.854 & -607.85328 & -607.905 \\
DAF & R4 & -607.865 & -607.852 & -607.85147 & 108.248 \\
\hline
\end{tabular}

\section{Geometry of DAF}

It was found that the carbon-carbon bond lengths in the oligomers are distorted in the charged states. The bond lengths of the neutral fluorene oligomer are not equivalent to the bond lengths of the charged fluorene oligomers. This is probably due to resonance distortion in the fluorene ring. This behavior varies in the charged states where the resonance is practically lost and slightly remaining in the center of the molecule(32). The partial loss of ring aromaticity forces some bond lengths to shorten or lengthen probably caused by the formation of quinoid-like structures. The different radical cations of fluorene substituted oligomer bond lengths are shown in Table. 3 .

Fig. 4. show the bond length between carbon-nitrogen are longer than the length bond between carbon-hydrogen and the bond length between nitrogen- hydrogen so the substituted on these positions are easier than another sites on the molecule.

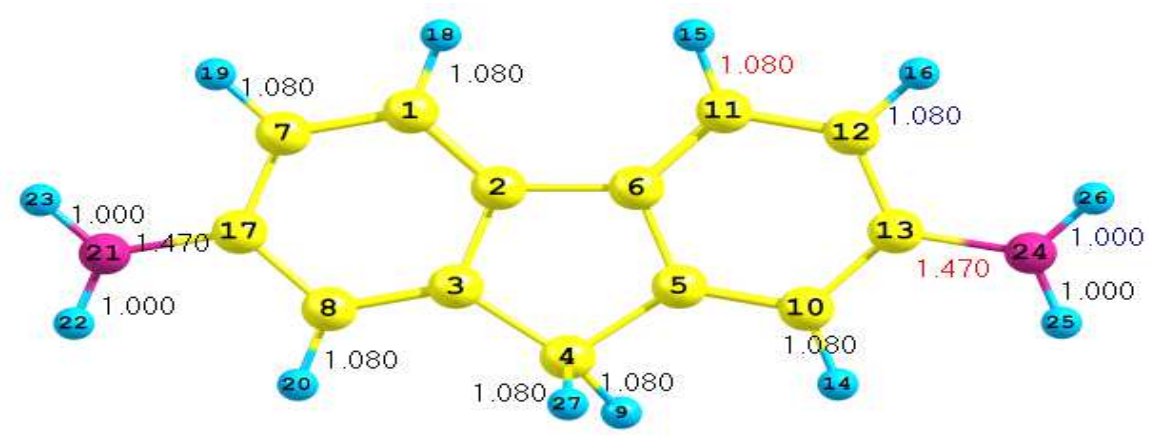

Figure 4. DAF plot of the molecular structure optimized at B3LYP/3-21G* and bond lengths and bond angles. 
Table 4. The Bond length (A) geometry of 2,7-DAF and DAF Radicals.

\begin{tabular}{lll}
\hline Bond length $(\AA)$ & DAF & R2 \\
\hline $\mathrm{R}(1,18)$ & 1.0846 & 1.0842 \\
$\mathrm{R}(7,19)$ & 1.0849 & 1.0845 \\
$\mathrm{R}(8,20)$ & 1.0857 & 1.0852 \\
$\mathrm{R}(10,14)$ & 1.0857 & 1.0862 \\
$\mathrm{R}(11,15)$ & 1.0846 & 1.0843 \\
\hline
\end{tabular}

\begin{tabular}{lll}
\hline Bond length $(\AA)$ & DAF & R2 \\
\hline $\mathrm{R}(12,16)$ & 1.0849 & 1.0824 \\
$\mathrm{R}(13,24)$ & 1.3802 & 1.3427 \\
$\mathrm{R}(17,21)$ & 1.3802 & 1.3735 \\
$\mathrm{R}(21,22)$ & 1.0105 & 1.0112 \\
$\mathrm{R}(21,23)$ & 1.0105 & 1.0112 \\
$\mathrm{R}(24,25)$ & 1.0105 & 1.0421 \\
\hline
\end{tabular}
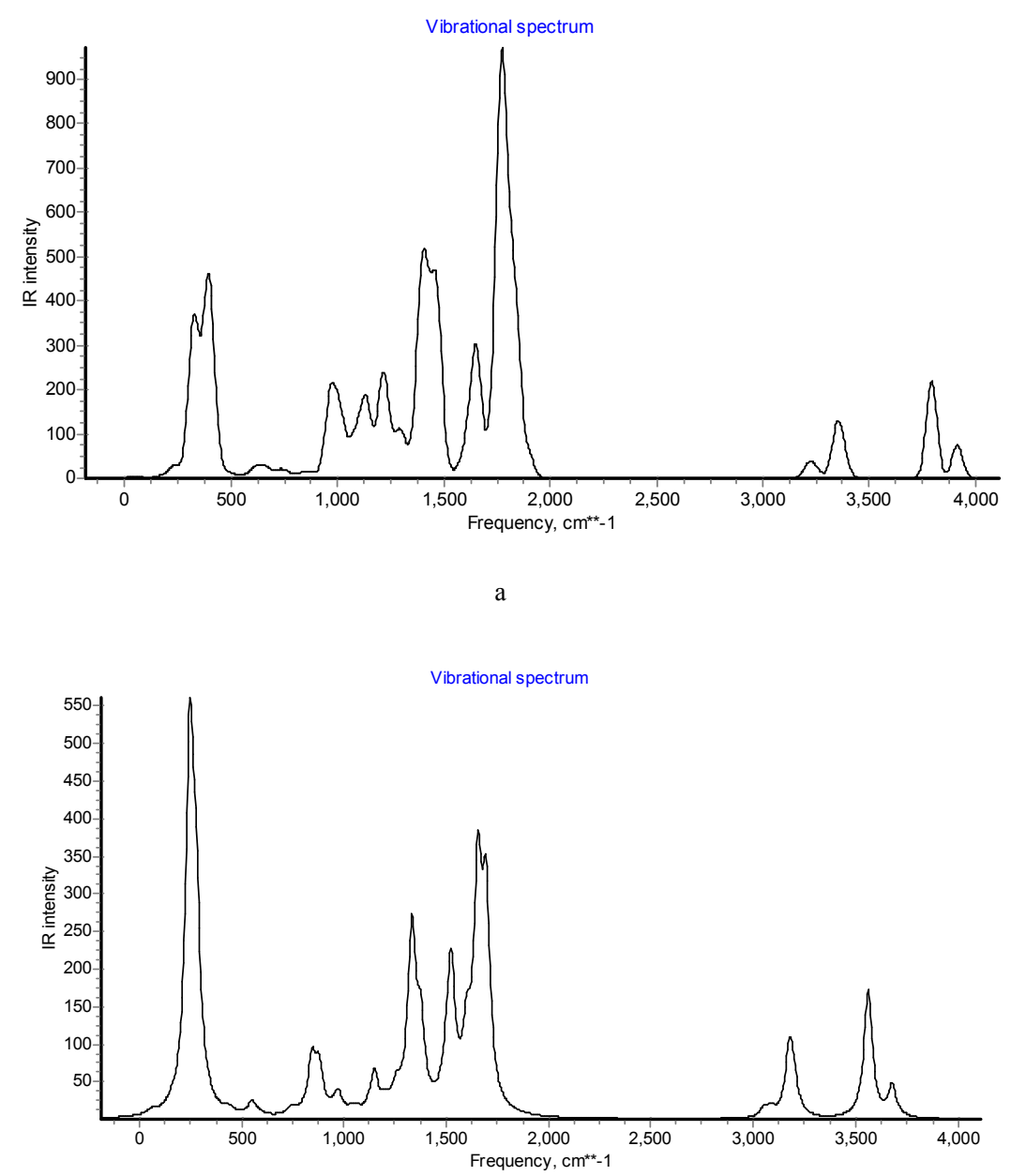

b

Figure 5. IR spectroscopy for a- DAF monomer, $b-D A F$ dimer at B3LYP/3-21G*.

\section{References}

[1] M. Yurtsever, E. Yurtsever, Chemical Physics Letters, 396, 2004, 424-428.

[2] D. Sazou, Ch. Georgolios, J. Electroanal. Chem, 429, 199781.

[3] S.H. Jin, M.Y. Kim, K. Lee, and Y.S. Gal, J. Am. Chem. Soc, 126, 2004, 2474.

[4] I. Markovich, D. Mandler, J. Electroanal. Chem, 500, 2001, 453.

[5] L.-W. Chong, Y.-L. Lee, T.-C. Wen, Thin Solid Films, 515, 2007, 2833.
[6] G. Zotti, S. Zecchin, B. Vercelli, A. Berlin, S. Grimoldi, L. Groenendaal, R. Bertoncello, M. Natali, Chem. Mater, 17, 2005,3681 .

[7] E.C. Venancio, C.A.R. Costa, S.A.S. Machado, A.J. Motheo, Electrochem. Comm, 3, 2001, 229.

[8] A.D. Becke, Phys. Rev. A38, 1988, 3089.

[9] A.D. Becke, J. Chem. Phys. 88, 1988, 2547.

[10] G. o. ppert-Mayer, M. Ann. Phys. 401, 1931, 273-291.

[11] W. Kaiser, C. G. B. Garrett, Phys. ReV. Lett, 7, 6, 1961, 229231.

[12] B. A. Reinhardt, Photo. Sci. News 4 (2), 1998, 21-34.

[13] S. Delysse, P. Raymond, Nunzi, J. M. Chem. Phys, 219, 1997, 341-351. 
[14] D. Beljonne, T. Kogej, S. R. Marder, Bre'das, J. L. Nonlinear. Opt. 21, 1999, 461-480.

[15] B. A. Reinhardt, L. L. Brott, S. J. Carlson, Dillard, A. G.; Bhatt, J. C. Kannan, R. Yuan, L. He, G. S.; P. N. Prasad, Chem. Mater. 10, 1998, 1863-1874.

[16] R. Vivas-Reyes, L. D. Mercado, J. Anaya-Gil, G. Andrés, E. Martinez, Theochem, 861, 2008. 137-141.

[17] J. Roncali, Chem. Rev. 97, 1997, 173-205.

[18] M. Del-Valle, F. Diaz, M. Bodini, G. Alfonso, G. Soto, E. Borrego, Polym. Int. 54, 2005, 526-532.

[19] J. Casanovas, E. Armelin, J. Iribarren, C. Aleman, F. Liesa, Polim. Ciên. Tecnol, 15, 2005, 239-244.

[20] G.M. Abou-Elenien, A.A. El-Maghraby, G. El-Abdallah, Synth. Metab, 146, 2004, 109.

[21] D.F. Acevedo, M.C. Miras, C.A. Barbero, J. Comb. Chem. 7, $2005,513$.

[22] Y.C. Liu, K.C. Chung, Synth. Metab. 139, 2003, 277.

[23] X. Li, Y. Kang, M. Huang, J. Comb. Chem. 8, 2006, 670-678.
[24] J. Chien, Polyacetylene: Chemistry, Physics, and Material Science, Academic Press, London, UK, 1984, 628.

[25] S. Yang, P. Olishevski, M. Kertesz, Synth. Metab. 141, 2004, 171-177.

[26] A. Violi, T. N. Truong, A. F. Sarofim, J. Phys. Chem. A 108, $2004,4846$.

[27] J. A. Miller, Pilling, M. J. Troe, J. Proc. Combust. Inst. 30, 2005,43 .

[28] J. A. Mulholland, Lu, M. Kim, D.-H. Proc. Combust. Inst. 28, 2000, 2593.

[29] D. Y. Kim, J. A. Mulholland, Wang, D. Violi, A. Combust. Flame, submitted for publication, 2005.

[30] D. Anna, A. Violi, A. Proc. Combust. Inst. 27, 1998, 425.

[31] A. Violi, A. F. Sarofim, T. N. Truong, Combust. Flame, 126, 2001, 1506 .

[32] M. J. Frisch, G. W. Trucks, H. B. Schlegel, G. E. Scuseria , M. A. Robb, J. R. Cheeseman, et al.Gaussian 03, revision C02. Wallingford CT: Gaussian Inc. 2004. 